\title{
High-density genotyping of 15 native Russian sheep breeds reveals genomic regions under selection related to domestication, acclimation and economically important traits
}

\author{
A.A. Yurchenko ${ }^{1}$, T.E. Deniskova ${ }^{2}$, A.V. Dotsev ${ }^{2}$, N.S. Yudin ${ }^{1,3}$, H. Reyer ${ }^{4}$, \\ K. Wimmers ${ }^{4}$, G. Brem², ${ }^{2}$, N.A. Zinovieva ${ }^{2}$, D.M. Larkin ${ }^{1,6 *}$ \\ ${ }^{1}$ Institute of Cytology and Genetics SB RAS, Novosibirsk, Russia \\ ${ }^{2}$ L.K. Ernst Federal Science Center for Animal Husbandry, Podolsk, Moscow region, Russia \\ ${ }^{3}$ Novosibirsk State University, Novosibirsk, Russia \\ ${ }^{4}$ Institute of Genome Biology, Leibniz Institute for Farm Animal Biology, Dummerstorf, Germany \\ ${ }^{5}$ Institute of Animal Breeding and Genetics, University of Veterinary Medicine, Vienna, Austria \\ ${ }^{6}$ Royal Veterinary College, University of London, London, UK \\ *e-mail:dmlarkin@gmail.com
}

Key words: sheep native breeds, selection, adaptation, genotyping, Russia

Motivation and Aim: There are over 1000 sheep breeds existing worldwide, many of which are adapted to local environments characterised by a variety of conditions: e.g. climates, parasite profiles, etc. Breeds were selected by humans for various traits, including wool quality/quantity, milk and meat production. Therefore, 9,000-11,000 years of domestication and breed design led to formation of local breeds that contain signatures of natural adaptation and artificial selection in their genomes. To identify genomic intervals that respond to selection and relate to adaptions to a variety of conditions found in Russian Federation we analysed 300 genomes of 15 Russian native sheep breeds.

Methods and Algorithms: A total of 312 representatives from 15 Russian native sheep breeds (ranging 20-24 samples per breed) were genotyped on the Illumina HD Ovine SNP Array, containing 600 M SNPs. The data were filtered in plink: --maf 0.05, --chr 1-26 and analysed with Admixture and PCA algorithms to identify groups of related breeds. We then performed detection of signatures of putative selection in the combined set of breeds and in two sets of related breeds separately using the hapFLK software.

Results: A total of 79 regions under putative selection ranging from $39 \mathrm{Kbp}$ to $5 \mathrm{Mbp}$ in size were identified in the combined set of 15 Russian breeds. The number of regions in two sets of related breeds was 104 and 64. A total of 556 genes were found within these intervals. The strongest signals of selection $\left(p\right.$-value $\left.<\mathrm{e}^{-23}\right)$ were detected near the genes: KIT, MC1R, MITF (coat color), PXFP2 (polled/horns), LCORL/ NCAPG (growth). We further detected strong signatures of selection in the regions containing genes known to be related to wool quality (PRLR, IRF2BP2), virial resistance (TMEM154), milk production (ABCG2,SOCS2), carcass (BMP2), and reproduction (VEGFA, NR3C1). In addition, candidate genes related to adaptation to cold stress/climate were identified in the selected regions, including: MMRN1, ADD1, ATF4, MCM8, and TSHR.

Conclusion: Multiple regions under putative selection were identified in the set of 15 Russian native sheep breeds forming a basis for future genomics-based selection and targeted breeding of Russian sheep.

Acknowledgements: This study was supported by the grants from the Russian Science Foundation (projects No. 16-14-00090 and 14-36-00039). 